\title{
Research on MCU Motor Control
}

\author{
Mujun Chen ${ }^{1, \text { a }}$, Jianjuan Zou ${ }^{2, b}$, Haiyan Qiu ${ }^{3, c}$, \\ ${ }^{1}$ Jiangxi Modern Polytechnic College, Nanchang, Jiangxi, China, 330095 \\ 2 Jiangxi Modern Polytechnic College, Nanchang, Jiangxi, China, 330095 \\ ${ }^{3}$ Jiangxi Modern Polytechnic College, Nanchang, Jiangxi, China, 330095 \\ aemail, ${ }^{\text {bemail, }}{ }^{\mathrm{c} e m a i l}$
}

Keywords: MCU, Motor, Control

\begin{abstract}
Motor control system has a simple structure, reliable operation and easy maintenance, etc., across the range of applications, including industrial sector. As a single-chip processor motor control system greatly reduces the cost, but also greatly simplifies the wiring of the PCB, PCB layout makes it easier. With the development of manufacturing technology and automation equipment needs, requires motor control system with high efficiency, high precision and high reliability, so a single-chip motor control system has become an inevitable trend.
\end{abstract}

\section{Introduction}

In recent years, with the single-chip performance continues to improve, which is widely applied to many fields of industry, agriculture, networking, communications, and in people's daily life. Not only to meet the many needs of applications, but also has high integration, powerful, fast, small size, low power consumption, easy to use, reliable and low cost characteristics.

As an important means motor electromechanical energy conversion, electric drive components are the basis of its power as a driving source in the production and life, it is widely used. There are many types of motors, different kinds of motors for different occasions. In the use of the motor required attention to the many problems, such as smooth speed DC motor and angle stepper motor control, rotation angle servo control, etc., where it relates to a motor control problem, only scientific and reasonable control motor, in order to make different motor play their superior performance. In the control method used in a variety of motor control MCU motor low cost, flexible control, precision accuracy. SCM in all types of motor control have played a huge role

Since the motor control system has been used in various areas of daily life, so the study of motor control system has been very active. Conventional stepping motor control system will consume a lot of electronic devices, commissioning and installation complexity, and change control program needs to be redesigned circuit, which will undoubtedly upgrade the control system is very unfavorable. With the software can control the motor control system based on single chip motor to overcome the disadvantages of conventional motor control system, so favored by the majority of researchers.

History and development SCM technology applications determines bound to have broad application prospects microcontroller-based motor control system; In addition, the current application needs smart home, intelligent also determines the direction of the control system, so that based on SCM motor control system has important practical significance.

\section{SCM and Control System}

According to the role of SCM in the control system, the control system can be divided into motor control system, control system based on SCM and the SCM system three categories of motor control.

Motor control system consists of a pulse controller, annular distributor, motor drive circuit and four major components, primarily issued by pulse controller pulse control signal, through the annular allocator, then transmitted to the drive circuit, power amplifier, in order to get enough 
power in a way that the motor operation.

SCM control system also consists of four parts, namely SCM system, drive system, the motor and the external circuit. SCM control system uses a combination of hardware and software methods for optimal control of the microcontroller. As the device has powerful features, so the design can also increase the number of peripheral circuits according to actual demand, for example, to control the motor operation (forward, reverse), stop, etc. via the keyboard.

SCM control system has many advantages, for some of the more complex control process and control high precision, you can write a program statement to achieve automatic control and precision control, the annular distributor in software, only need to set the microcontroller, for the same species circuit configuration and can drive a variety of motor control, thereby greatly improving the versatility and flexibility with the motor circuit microcontroller interface; and single-chip peripheral circuits such as a keyboard so that the combination of the circuit, greatly improved interactivity. With digital control system in the microcontroller as the processor, and analog control systems compared to control high precision, no zero drift control system. Digital control system uses software to control, to achieve high-speed, high-precision requirements. For system upgrades, system commissioning or change the control mode, simply change the program. With the rapid development of electronic technology, the demand for the motor control system is also rising, there has been dedicated to motor control microcontroller, mainly inside the microcontroller adds high-speed converters, the output terminal and capture functions. Analog signal into the microcontroller, the first to go through the high-speed converter to ensure sampling accuracy by capturing function to measure its frequency. SCM gradually improve work efficiency, and some can even be compared with the single-chip DSP, microcontroller faster, more easily qualified for complex algorithms, real-time tracking system.

\section{Control System Design}

For motor control system, most of the design focused on regulating the electromagnetic torque of the motor as needed to control the motor speed. A current proportional to the magnetic moment and the windings on the motor control system produced by winding, and winding current from the winding voltage to control the voltage on the winding originated in inverter unit, controlled by the drive switch duty cycle. Thus, the winding voltage can be adjusted by varying the duty cycle, thereby regulating the magnetic torque, and ultimately to control the speed.

To improve the performance parameters of the motor control system, in most cases the motor control system uses a closed loop motor control mode, when the most common closed-loop motor speed control system for closed-loop motor control system [2]. Adjust the speed of the most effective way is to adjust the electric drive current by adjusting the electric drive dynamic response speed of the current can get a high-performance current control loop in the construction of the motor control system is critical. In order to achieve the speed, respectively, feedback and current role, the system can be set up through a series arrangement respectively speed regulator and current regulator, the current regulator operates in the inverter output and the speed regulator acts on the current regulator input eventually adjust the motor speed to achieve the effect. From the closed-loop structure, the outer ring to adjust the speed of the outer, inner current regulation loop included. In the double-loop (speed, current average closed loop) motor control system, a single closed loop motor control system speed advantage is retained, to ensure that the speed with changes in demand, which is different from the current regulator that provides an output size It determines the maximum current. The role of the current regulator is a current value with a given change, when you start to ensure maximum torque capable of fast start, and thus play a role in immunity.

During the study the electronic control system, the principles of control system simulation is necessary, the simulation can guide motor control system hardware and software design. The most commonly used simulation software in MATLAB simulink, including dynamic system modeling, simulation, and comprehensive analysis of the environment. Next article describes the emulated hardware design microcontroller-based motor control systems, software design and control system. Motor control system hardware design is divided into two main control circuit and a power circuit 
module, were designed PCB board, such control panel design is more convenient, can be used in various environments. Motor control system hardware structure to use more pay, straight, cross-conversion mode, the phase current signal and the rotor position signals to the motor control circuit by the power board, the role of the power board interface circuit is the use of optical isolation circuit to the control circuit the signal is transmitted to the drive circuit. Wherein the power circuit design in the whole motor control system design is very important, it is the source of energy for the entire motor control system. In the design of the microcontroller hardware-based motor control system, generally it needs to be considered part of the rectifier circuit, a power driver circuit, detecting circuit and printed circuit board electromagnetic compatibility and so on.

\section{SCM DC Motor}

DC to DC can be converted to mechanical energy, now used more for DC motor armature rotating pole field winding and surrounded by the core consisting of a stator with armature windings and the armature core constituting the DC converter unit combination the motor rotor. DC motor has the following advantages: wide speed range, smooth and easy to adjust; overload, starting, braking torque; easy to control, high reliability; less energy loss when adjusted.

Between the above advantages in situations that require high speed, such as rolling mills, propeller, trams, cranes, textiles, etc., to obtain a wide range of DC motor applications. Most MCU control DC motor is used to adjust the speed of a DC motor, PWM speed control is a reliable solution.

PWM DC motor speed control principle: PWM control is pulse width modulation technology, through a series of pulse width mediation to effectively obtain the desired waveform. By controlling the MCU I / O port output different duty cycle of the PWM waveform can control the motor speed. High motor is turned on, low motor off, control the motor, the greater the duty cycle, the faster the entire period when the whole is high, one hundred percent duty cycle, the fastest.

When the MCU I / O port output PWM signal can use the following three ways:

Use software delay. When high delay time to time, for I / O port becomes a low level negated, then the delay; when low delay time to time, and then the I / O port level negated, so cycle of the PWM signal can be obtained.

Use of the timer, the control method as above, but with a timer to do the reverse order to achieve the level of precision delay.

The use of built-in microcontroller PWM controller. STC12, AVR, PIC and other microcontroller has this feature, but the 51 series of microcontrollers can not.

DC motor drive for general use Darlington, or ASIC, small motor case under a simple circuit transistor can also be used to build other basic components.

\section{SCM Control Stepper Motor}

Stepper motors are three-wire, five-wire and six-wire, but its control method are the same, every pulse signal current to drive. If one revolution requires 100 to the excitation pulse, each excitation signal enables the stepper motor forward 3.6 degrees, and the angle of rotation is proportional to the number of pulses. Stepper motor reversing the order by the pulse generator to control.

Excitation mode Stepper motors have full step and half step excitation field, the field is divided into a full-step phase excitation and two-phase excitation, half step excitation is one hundred twelve phase excitation. Suppose one revolution requires 100 pulse excitation signal can be calculated for each excitation signal enables the stepper motor rotates 3.6 degrees.

\section{Microprocessor Controlled Servo}

General servos have power line VCC, ground GND and the control signal line three lines. Wherein the control signal is received by the microcontroller line input PWM signal, PWM signal into the servo signal conditioning chip to control the position of the steering gear. It has an internal 
reference circuit generates a cycle of $20 \mathrm{~ms}, 1.5 \mathrm{~ms}$ width of the reference signal, and the DC voltage and paranoia potentiometer voltage then obtained by comparing the voltage difference between the output obtained. Finally, the voltage difference between the positive and negative outputs to the motor driver chip motor positive decision.

In practice, you can also use a microcontroller to control multiple servos. If the microcontroller 51, you need to repeatedly interrupt the timer count for software to control them too much trouble, but you can use AVR microcontroller to generate multi-channel PWM signal.

\section{Conclusion}

In addition to choosing the right microcontroller based on demand, but also pay attention to the motor coupled to the motor circuit control in practical applications, different types of motor control is small can be used to build transistors and other circuits, control of the motor is generally used in a long time Darlington or a dedicated motor driver chip, such as L298N eight motor control system, the user can edit the reasonable control program according to different needs, this approach is to highlight the advantages of low cost, flexible control for a wide range in the motor the control system plays a major role.

\section{References}

[1] Jing Yang, Li Jingyuan. Machine Tool Electric Apparatus, Vol. 6 (2004) No 53, p.25-26

[2] Wang Qunyong. Coal Technology, Vol. 12 (2005) No 27, p.74-76

[3] Wang Wei, Jing Jianfen, Electrotechnical, Vol. 30 (2004) No 19, p.144-145

[4] Zhao Fengxian. Instrument Technique and Sensor, Vol. 29 (2008) No 27, p.21-23

[5] Wang Zongpei, Sun Jing. Electrical Technology Magazine, Vol. 8 (2003) No 27, p.57-60 